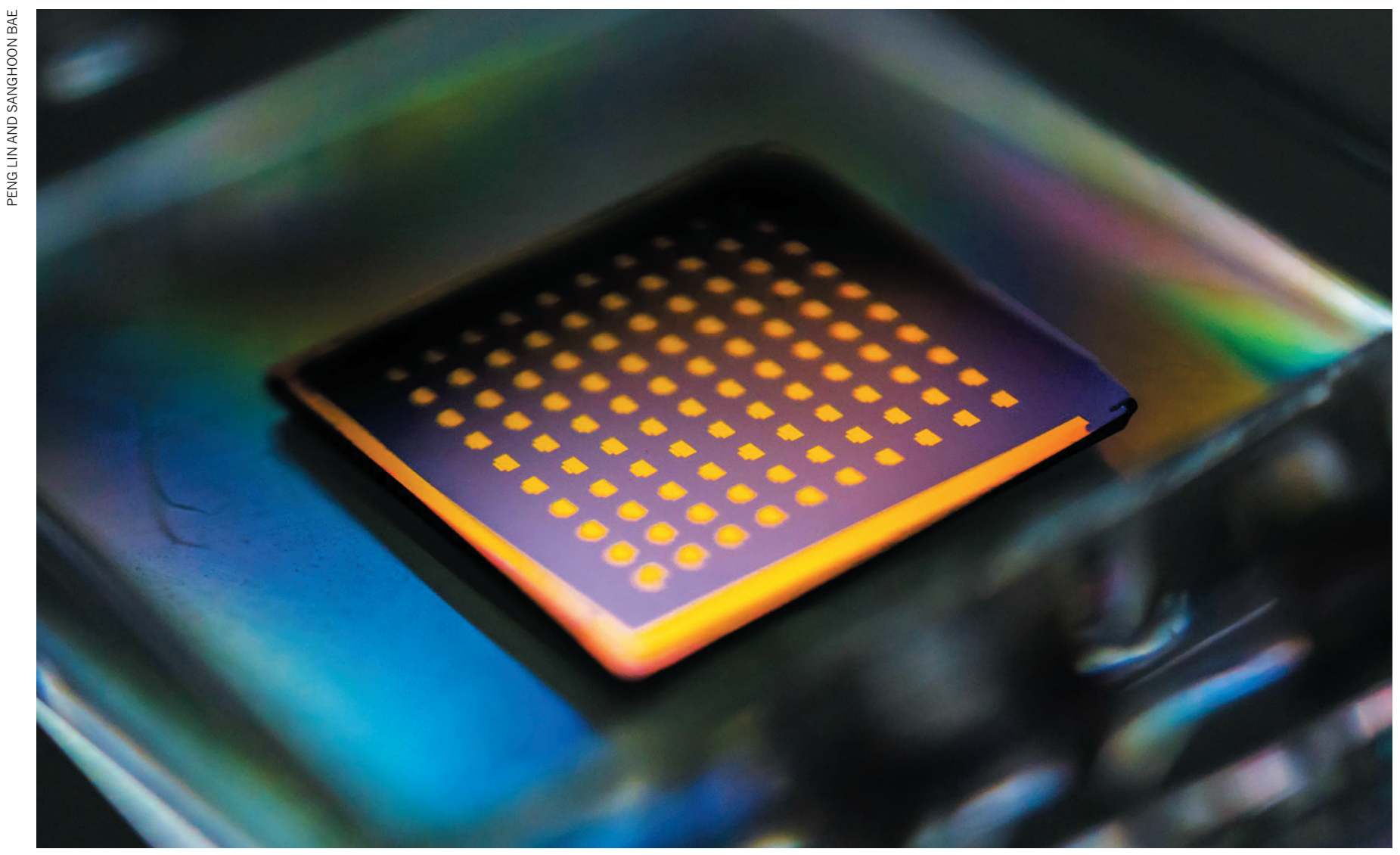

Chips made from stacks of transistors just one molecule thick might power the next generation of computers.

\title{
How 2D semiconductors could extend Moore's law
}

\section{Incredibly thin transistors could deliver even more powerful computers if three research challenges can be solved, argue Ming-Yang Li and colleagues.}

$\mathrm{T}$ The number of components in electronic circuits has doubled every

two years since the 1960 s - a trend known as Moore's law. Transistors have shrunk so that ever more can fit on a silicon chip. But now, silicon transistors are reaching their physical limit. Radically different types of materials and devices are needed to realize the potential of the next generation of computers.

The problem is common to all transistors based on bulk (3D) semiconductors, including those made of germanium, indium gallium arsenide and indium phosphide. Electrons find it hard to travel through channels nanometres in thickness. Imperfections in the surfaces of the channels scatter charges, slowing their flow.

But 2D materials could enable transistors to keep shrinking. These are crystalline sheets of atoms, one layer thick. Because they have only a limited 'vertical' dimension, and flat surfaces free from defects, electrons are less prone to scattering and charges can flow relatively freely through them. Promising materials include transition-metal dichalcogenides (such as tungsten diselenide and molybdenum disulfide, $\mathrm{MoS}_{2}$ ).

However, this research is still at an early stage. For these materials to meet industry needs in practical devices, three fundamental research challenges must be resolved.

\section{THREE CHALLENGES}

Predicting properties accurately. First, we need to find materials that are suitable for $2 \mathrm{D}$ transistors. There are more than 1,000 candidates, and their electronic properties vary wildly. It is quicker to predict electronic properties theoretically than to weed them out experimentally, but it is difficult to accurately derive the behaviour of a material in this way. The energies of electrons depend on the specific nature and arrangement of atoms in the crystal lattice, and this is often more complicated in real materials than in idealized models. Interactions between electrons and atoms must be accounted for, and approximations add uncertainty.

And experiments have found that electrons are not as mobile as expected within $2 \mathrm{D}$ sheets. This is due to electron scattering, but the processes involved are poorly understood. For example, how do vibrations of the lattice slow electrons? Adjacent layers of materials, such as the substrate and protective films, also have an influence, as do nearby charges and imperfections. Physicists need to consider all of these factors.

Growing and testing samples. Promising candidates need to be tested experimentally. It is important that the samples are of high 


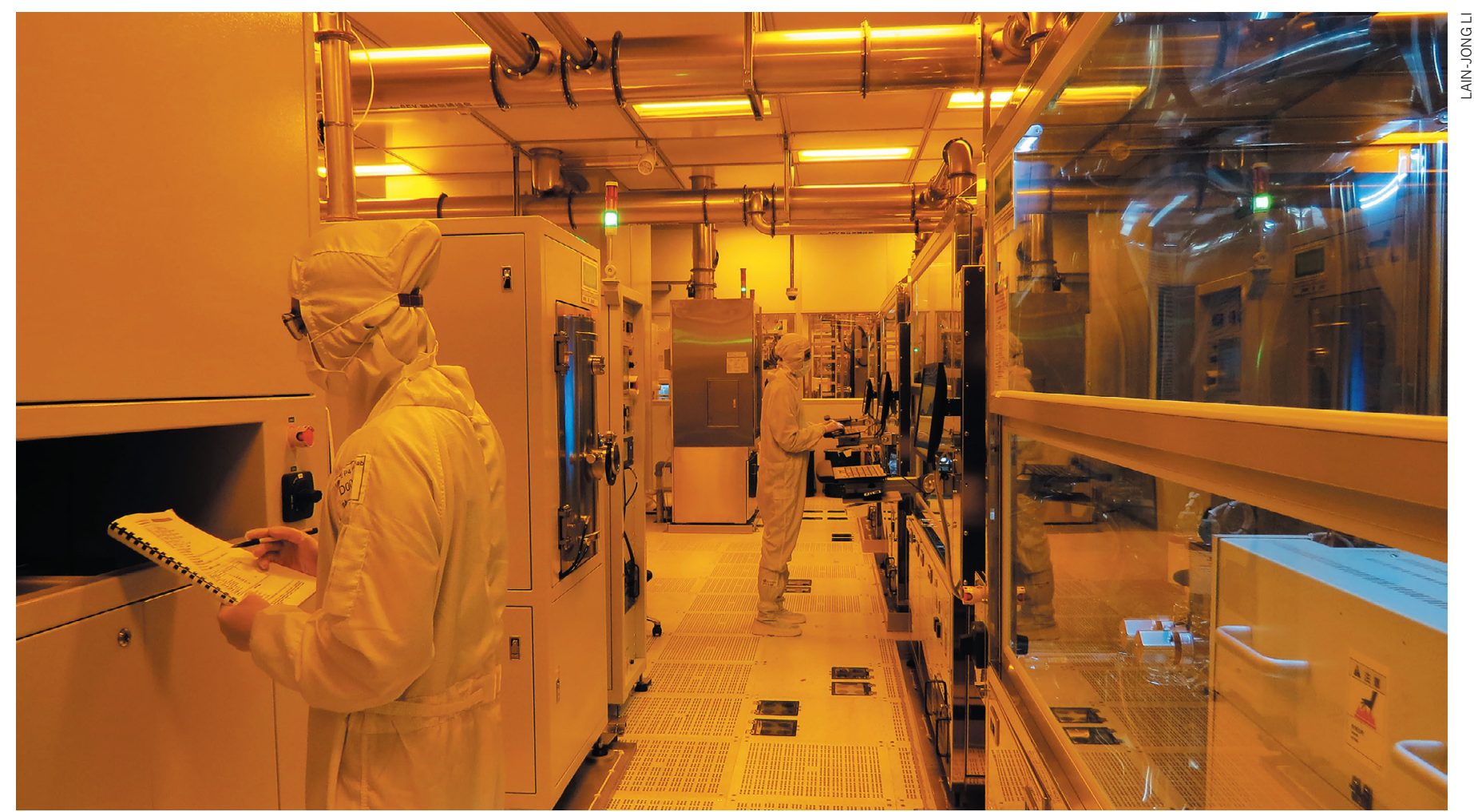

Researchers in the Semiconductor Materials Advanced Research and Technology laboratory at the Taiwan Semiconductor Manufacturing Company in Hsinchu.

quality and are large enough to incorporate into state-of-the-art transistors and simple benchmark circuits. Defects and grain boundaries affect charge flow ${ }^{1}$. So researchers need to learn how to grow uniform sheets of $2 \mathrm{D}$ materials with perfectly aligned crystals. They also need to control the thickness of layers to adjust the electronic properties.

There are two ways to make 2D sheets of crystals. The better option is to grow them by depositing atoms from vapours in a vacuum chamber. They can also be formed through exfoliation, by either peeling the layers off mechanically or shedding them in solution. Peeling results in fewer lattice defects than shedding, but the amounts produced are too small for practical devices.

When forming crystals, the substrate affects their growth. 3D materials bond tightly to the underlying layer and follow its atomic geometries closely. By contrast, 2D materials are only loosely attached to the substrate, but are nevertheless influenced by it. For example, $\mathrm{MoS}_{2}$ crystals grown on sapphire can follow two orientations ( 0 degrees and 60 degrees). Other materials form lessdirected grains with boundaries similar to the joints in a jigsaw. Some spots end up being more than one molecular layer thick.

2D materials need to be made chemically and thermally stable. For example, phosphorene and indium selenide degrade in ambient air within hours ${ }^{2,3}$. Adding a protective film, such as an oxide, can help to preserve the material, but this adds another interface to consider.

Ultimately, transistors need to be reliably manufactured in large quantities. Today, the industry typically uses silicon substrates and silicon dioxide insulators, but these cannot be used for oriented growth, and their electrical quality can degrade at the high temperatures $\left(600-1,000^{\circ} \mathrm{C}\right)$ needed to deposit atoms from vapours. Thus, $2 \mathrm{D}$ materials would have to be grown on expensive silicon carbide or sapphire substrates, which can withstand such heat, and then transferred onto silicon substrates for integration into the device. Molecular beam epitaxy (spraying beams of atoms in a vacuum) is another method for growing $2 \mathrm{D}$ materials at lower temperatures (below $300^{\circ} \mathrm{C}$ ), but this is slow: samples might take days to grow. Yield and reproducibility must also be improved.

Assessing device performance. The performance of different 2D materials should be assessed within a common framework. Measurements reported in different settings, or even labs, might be inconsistent. The precise composition and structure of substrates, sandwiching layers, electronic contacts and fabrication processes all matter. And each type of device brings its own demands. For example, a transistor in a logic switch must turn on and off sharply, and an energy-efficient transistor must operate at low voltage.

Academic and industrial researchers should collaborate to devise measurement standards, similar to those set for solar-cell efficiency assessments by the US National Renewable Energy Laboratory. These should stipulate, for example, the structure of the device, criteria for metal contacts and procedures for measurement.

\section{COLLABORATION AND FUNDING}

The nearly half-a-trillion-dollar semiconductor-chip industry should invest in solving these research problems; governments, ditto. The applications of graphene, the best-known 2D material, and methods for its mass production are being developed through the European Commission's $€ 1$-billion (US\$1.1-billion) Graphene Flagship research programme. But other $2 \mathrm{D}$ materials are still largely in the academic phase.

2D materials should play a larger part in electronics programmes exploring materials for logic, memory and connections. One such effort is the 2D Atomic-layer Research and Engineering programme at the US National Science Foundation. More groups, such as the Semiconductor Research Corporation, a non-profit consortium based in Durham, North Carolina, should set up programmes to accelerate the development of $2 \mathrm{D}$ materials.

It's too early to say whether $2 \mathrm{D}$ semiconductors will transform transistors. But they are one of the best candidates we have to extend Moore's law.

Ming-Yang Li, Sheng-Kai Su, H.-S. Philip Wong and Lain-Jong Li are with Corporate Research at the Taiwan Semiconductor Manufacturing Company, Hsinchu, Taiwan. e-mails: ljliv@tsmc.com; hspwong@tsmc.com

1. Ly, T. H. et al. Nature Commun. 7, 10426 (2016).

2. Pei, J. et al. Nature Commun. 7, 10450 (2016).

3. Chang, H.-C. et al. Small 14, 1802351 (2018). 